\title{
Studying the Characteristics of Algebraic Curve Behavior For Nonstandard Method
}

\author{
Hind Y. Saleh \\ College of Basic Education, University of Duhok, Duhok, Kurdistan Region - Iraq
}

\begin{abstract}
This research aims to study some algebraic curve behaviors in monad (halo) of the regular and irregular points. For this, the Robinson ideas can be used for limited points. Also the geometric tangent at this limited point and the analysis of the algebraic curve around the same point will be studied. Concepts of nonstandard analysis given by Robinson and its axiomatic by Nelson have been used. In this research, we have obtained a state where the point of origin is a regular point and concave is as illustrated in (5). So the curve has connection in this equation (6). There is a second state where the point of origin is an abnormal point of the curve as illustrated in (11). Finally, it has a concave that is calculated in the equation (29).

KEYWORDS : Monad, Infinitesimal, Limited, Shadow, Regular and Irregular points.
\end{abstract}

\section{INTRODUCTION}

Nonstandard Analysis, as is the case with mathematics itself, can be applied to many domains so as to illustrate this by means of examples is the object of (Diener F. and Diener M . 1995). In 1961, Robinson showed that there exists a proper extension of the field of real numbers which, in a certain sense, has the same formal properties $\mathrm{R}$ and Archimedean. In 1965, he introduced the concept of external sets (Robinson A. 1970). Today, there are several versions of nonstandard Analysis structure made by many mathematicians like K. Stroyan, and W. Luxemburg (Rosinger E. E. 2004), E. Nelson(Nelson E. 1977) , K. Hrbacek (Habacek K. 1979) , C. Henson(Henson C. W. 1997) , E. Rosinger (Rosinger E. E. 2004) , ... etc. In this work, we have used Nelson's nonstandard construction, based on a theory called internal se theory IST which is constructed on the axiomatic set theory of ZFC (Zermelo-Fraenckel set theory with Axiom of Choice) (Nelson E. 1977), and with a new undefined predicate "standard". In fact, the curve behavior and the tangent of curves at any point form an important subject in mathematics. So, the study of this issue by using nonstandard methods is very interesting

Academic Journal of Nawroz University

(AJNU) Volume 7, No 3 (2018).

Received 1 March 2018;

Regular research paper : Published 20 June 2018

Corresponding author's e-mail : Hind.saleh@uod.ac

Copyright (02018 Hind Y. Saleh.

This is an open access article distributed under the

Creative Commons Attribution License. because it gives us new results. The study includes the algebraic behavior of this curve (say $C$ ) in monad of their points., and the tangent of this curve at regular and irregular points geometrically. Throughout this work, the following definitions are needed.

\section{Definitions}

2.1 A real number $x$ is said to be unlimited (infinity large) if $|x|>r$ for all positive standard real numbers $r$, otherwise it is called limited.(Daivs M. 1977),(Diener M. and Lobry C. 1995) and (Diener F. and Reeb G. 1989).

2.2 A real number $x$ is said to be infinitesimal if $|x|<r$ for all positive standard real numbers $r$. (Diener M. and Lobry C. 1995),(Diener F. and Diener M . 1995), (Nelson E. 1977) and (Stroyan K. D. and Luxemburg W. A. 1976). 2.3 A real number $x$ is said to be appreciable if $x$ is limited but not infinitesimal. (Stroyan K. D. and Luxemburg W. A. 1976) and (Vath M. 2007)

2.4 Two real numbers $x$ and $y$ are said to be infinity closed (near) if $x-y$ is infinitesimal and denoted by $x \simeq$ $y$.(Daivs M. 1977),(Diener F. and Reeb G. 1989),(Henson C. W. 1997) and (Nelson E. 1977).

2.5 If $x$ is a limited number in $\mathbb{R}$, then it is infinity close to a unique standard real number, which is called standard part or shadow of $x$ and dented by ${ }^{\circ} x$ or st $(x)$. (Diener M. and Lobry C. 1995),(Diener F. and Reeb G. 1989) (Diener F. and Diener M . 1995) and (Nelson E. 1977)

2.6 The set of real numbers which are infinity close to a real number $x$ is called monad or halo of $x$, and denoted by $m(x)$ or $h a l(x)$. So the set of real numbers which are infinity close to 0 is called monad or halo of 0 , and denoted by $m(0)$ or hal(0).(Diener F. and Diener M . 1995), (Robinson A. 1970) and (Rosinger E. E. 2004). 
2.7 For any real number $a$, the set of all real numbers $x$ such that $x-a$ limited is called galaxy of $a$, and denoted by $\operatorname{gal}(a)$. The set of all limited real numbers is called principal galaxy and denoted by Gal (Daivs M. 1977), (Diener M. and Lobry C. 1995), (Diener F. and Reeb G. 1989) and (Robinson A. 1970).

2.8 Let $\beta$ be a nonzero real number and $\in \mathbb{R}$, we define $\beta-\operatorname{gal}(x)=\left\{y \in \boldsymbol{R}: \frac{y-x}{\beta}\right.$ is limited $\}$. (Diener M. and Lobry C. 1995), (Diener F. and Diener M . 1995) and (Robinson A. 1970).

The problem : Consider $C$ as a standard curve after the conversion of the compound as follows

$$
f(x, y)=a_{0} x^{3}+a_{1} y^{3}+a_{2} x^{2} y+a_{3} x y^{2}+a_{4} x^{2}+a_{5} y^{2}
$$$$
+a_{6} x y+a_{7} x=0 \ldots \ldots \text { (1) }
$$

Suppose $\mathrm{M}$ is a point that belongs to the monad of zero, a standard base is given by :

$v_{1}=\left[\begin{array}{l}\alpha \\ \beta\end{array}\right]$ and $v_{2}=\left[\begin{array}{c}\beta \\ -\alpha\end{array}\right]$

Where $\varepsilon_{1}$ and $\varepsilon_{2}$ are infinitesimal (in monad of zero), then

$$
M=\varepsilon_{1} v_{1}+\varepsilon_{1} \varepsilon_{2} v_{1} .=\left[\begin{array}{c}
\varepsilon_{1} \varepsilon_{2} \\
\varepsilon_{1}
\end{array}\right]
$$

Assume $M$ is a point on the curve $C$ and the equation $f(M)=0$, using the shadow gives $a_{7} x=0$.

\section{Main Results :}

\section{Case 1 :}

If $a_{7} \neq 0$, in this case the original point will be a regular point, and $\alpha=0$, choose $v_{1}$ as the tangent at original point of the carve, i.e. $v_{1}=\left[\begin{array}{l}0 \\ 1\end{array}\right]$ and $v_{2}=\left[\begin{array}{l}1 \\ 0\end{array}\right]$, this gives the infinitesimal vector as

$M=\left[\begin{array}{c}\varepsilon_{1} \varepsilon_{2} \\ \varepsilon_{1}\end{array}\right]$.

Theorem 3.1 : if $a_{7} \neq 0$, then the concave of the curve $C$ at original point is given by the formula

$k=\left|\frac{\varepsilon_{2}}{\varepsilon_{1}}\right|=\left|\frac{a_{5}}{a_{7}}\right|$

Proof : By using the vector $M$, and equation $f(M)=0$, we have

$$
f(M)=a_{0} \varepsilon_{1}^{3} \varepsilon_{2}^{3}+a_{1} \varepsilon_{1}^{3}+a_{2} \varepsilon_{1}^{3} \varepsilon_{2}^{2}+a_{3} \varepsilon_{1}^{3} \varepsilon_{2}+a_{4} \varepsilon_{1}^{2} \varepsilon_{2}^{2}+a_{5} \varepsilon_{1}^{2}
$$$$
+a_{6} \varepsilon_{1}^{2} \varepsilon_{2}+a_{7} \varepsilon_{1} \varepsilon_{2}=0 \ldots \ldots \text { (3) }
$$

Divide (3) by $\varepsilon_{1}^{2}$ to get the form

$a_{0} \varepsilon_{1} \varepsilon_{2}^{3}+a_{1} \varepsilon_{1}+a_{2} \varepsilon_{1} \varepsilon_{2}^{2}+a_{3} \varepsilon_{1} \varepsilon_{2}+a_{4} \varepsilon_{2}^{2}+a_{5}+a_{6} \varepsilon_{2}+$ $a_{7}\left(\frac{\varepsilon_{2}}{\varepsilon_{1}}\right)=0$

Taking the shadow for (4), yields

$$
\left(\frac{\varepsilon_{2}}{\varepsilon_{1}}\right)=\left(-\frac{a_{5}}{a_{7}}\right)
$$

Therefore the absolute value of both sides of (5) will complete the proof.

Theorem 3.2 : If $a_{7} \neq 0$, then the curve has the following connection :

$x=x(t)=\frac{-a_{5}}{a_{7}} t^{2}+\left(\frac{-a_{1}}{a_{7}}+\frac{a_{5} a_{6}}{\left(a_{7}\right)^{2}}\right) t^{3}+\cdots$

$y=y(t)=t$

Proof :

Equation (5) can be rewritten as $\varepsilon_{2}=\varepsilon_{1}\left(\frac{-a_{5}}{a_{7}}+\varepsilon_{1}^{\prime}\right)$,

where $\varepsilon_{1}^{\prime} \simeq 0$

The value $\varepsilon_{2}$ can be substituted in (4) to yield

$$
\begin{aligned}
f(M)=a_{0} \varepsilon_{1}^{4}\left(\frac{-a_{5}}{a_{7}}\right. & \left.+\varepsilon_{1}^{\prime}\right)^{3}+a_{1} \varepsilon_{1}+a_{1} \varepsilon_{1}^{3}\left(\frac{-a_{5}}{a_{7}}+\varepsilon_{1}^{\prime}\right)^{2} \\
& +a_{3} \varepsilon_{1}^{2}\left(\frac{-a_{5}}{a_{7}}+\varepsilon_{1}^{\prime}\right)+a_{4} \varepsilon_{1}^{2}\left(\frac{-a_{5}}{a_{7}}+\varepsilon_{1}^{\prime}\right)^{2} \\
& +a_{5}+a_{6} \varepsilon_{1}\left(\frac{-a_{5}}{a_{7}}+\varepsilon_{1}^{\prime}\right)+a_{7}\left(\frac{-a_{5}}{a_{7}}+\varepsilon_{1}^{\prime}\right) \\
& =0
\end{aligned}
$$

Diving both sides of (7) by $\varepsilon_{1}$,

$$
\begin{aligned}
& f(M)=a_{0} \varepsilon_{1}^{3}\left(\frac{-a_{5}}{a_{7}}+\varepsilon_{1}^{\prime}\right)^{3}+a_{1}+a_{1} \varepsilon_{1}^{2}\left(\frac{-a_{5}}{a_{7}}+\varepsilon_{1}^{\prime}\right)^{2}+ \\
& a_{3} \varepsilon_{1}\left(\frac{-a_{5}}{a_{7}}+\varepsilon_{1}^{\prime}\right)+a_{4} \varepsilon_{1}\left(\frac{-a_{5}}{a_{7}}+\varepsilon_{1}^{\prime}\right)^{2}+\frac{a_{5}}{\varepsilon_{1}}+a_{6}\left(\frac{-a_{5}}{a_{7}}+\varepsilon_{1}^{\prime}\right)+
\end{aligned}
$$$$
\frac{a_{7}}{\varepsilon_{1}}\left(\frac{-a_{5}}{a_{7}}+\varepsilon_{1}^{\prime}\right)=0
$$

The shadow of (8) gives

$$
\begin{gathered}
{ }^{\circ} a_{1}+{ }^{\circ}\left(\frac{a_{5}}{\varepsilon_{1}}\right)+{ }^{\circ} a_{6}\left(\frac{-a_{5}}{a_{7}}+\varepsilon_{1}^{\prime}\right)+{ }^{\circ}\left(\frac{a_{7}}{\varepsilon_{1}}\right)^{\circ}\left(\frac{-a_{5}}{a_{7}}+\varepsilon_{1}^{\prime}\right) \\
=0 \ldots \ldots \ldots \ldots \ldots \ldots \text { (9) }
\end{gathered}
$$

Therefore, the point $\mathrm{M}$ can be written as

$$
\begin{aligned}
& M=\left[\begin{array}{c}
\varepsilon_{1} \varepsilon_{3} \\
\varepsilon_{1}
\end{array}\right] \\
& =\left[\begin{array}{c}
\frac{-a_{5}}{a_{7}} \varepsilon_{1}^{2}+\left(\frac{-a_{1}}{a_{7}}+\frac{a_{5} a_{6}}{\left(a_{7}\right)^{2}}\right) \varepsilon_{1}^{2}+\varepsilon_{1}^{\prime \prime} \varepsilon_{1}^{3} \\
\varepsilon_{1}
\end{array}\right]
\end{aligned}
$$

where $\varepsilon_{1}^{\prime \prime}$ in the monad of zero.

This gives one of the connections for the curve $C$.

\section{Case 2 :}

When $a_{7}=0$, the original point is an irregular point for the curve $C$ from the equation $f(M)=0, M=(\alpha, \beta)$ and in the equation (1) if

$a_{4} \alpha^{2}+a_{6} \alpha \beta+a_{5} \beta^{2}=0$

The result depends on $\Delta=a_{6}^{2}-4 a_{4} a_{5}$.

i- if $\Delta<0$, the Cone of tangents at the original point is an empty set. For this, the original point will be isolated from the curve $C$.

ii- if $\Delta=0$, we have a unique vector in a cone of tangents and get curve in double points called private curves.

Theorem 3.3 : If $\Delta=0$, the curve will be an abnormal curve, and allows partial connection at original point which is the relative connection point

$$
k=\left|\frac{\varepsilon_{1}}{\varepsilon_{2}^{1 / 2}}\right|=\left|\sqrt{\frac{\alpha_{1}}{\alpha_{4}}}\right| \ldots \ldots \ldots
$$

\section{Proof :}

If $\Delta=0$, the coordinates of the curve $C$ in (3.1) can be written as

$$
\begin{gathered}
f(x, y)=a_{0} x^{3}+a_{1} y^{3}+a_{2} x^{2} y+a_{3} x y^{2}+a_{4} x^{2} \\
=0 \ldots \ldots \ldots \ldots \ldots \ldots \ldots \ldots \ldots \ldots \ldots \ldots \\
f\left(\varepsilon_{1} \varepsilon_{2}, \varepsilon_{1}\right)=\alpha_{0} \varepsilon_{1}{ }^{3} \varepsilon_{2}^{3}+\alpha_{1} \varepsilon_{1}{ }^{3}+\alpha_{2} \varepsilon_{1}{ }^{3} \varepsilon_{2}^{2}+\alpha_{3} \varepsilon_{1}{ }^{3} \varepsilon_{2} \\
+\alpha_{4} \varepsilon_{1}{ }^{2} \varepsilon_{2}^{2}=0 \ldots \ldots(13)
\end{gathered}
$$

Where $a_{1} \neq 0$, and $a_{4} \neq 0$, except that the curve become span. Therefore, the same expression in (2), diving (13) 
by will yield $f(M)=\alpha_{0} \varepsilon_{1} \varepsilon_{2}^{3}+\alpha_{1} \varepsilon_{1}+\alpha_{2} \varepsilon_{1} \varepsilon_{2}^{2}+\alpha_{3} \varepsilon_{1} \varepsilon_{2}+\alpha_{4} \varepsilon_{2}^{2}$
$=0$

Now, we have

$\varepsilon_{1}\left(\alpha_{0} \varepsilon_{2}^{3}+\alpha_{1}+\alpha_{2} \varepsilon_{2}^{2}+\alpha_{3} \varepsilon_{2}\right)=-\alpha_{4} \varepsilon_{2}^{2}$

$\varepsilon_{1}=\frac{-\alpha_{4} \varepsilon_{2}^{2}}{\alpha_{0} \varepsilon_{2}^{3}+\alpha_{1}+\alpha_{2} \varepsilon_{2}^{2}+\alpha_{3} \varepsilon_{2}} \ldots \ldots$.

Satisfying in (2), will yields

$M=\left[\begin{array}{c}\frac{-\alpha_{4} \varepsilon_{2}^{3}}{\alpha_{0} \varepsilon_{2}^{3}+\alpha_{1}+\alpha_{2} \varepsilon_{2}^{2}+\alpha_{3} \varepsilon_{2}} \\ \frac{-\alpha_{4} \varepsilon_{2}^{2}}{\alpha_{0} \varepsilon_{2}^{3}+\alpha_{1}+\alpha_{2} \varepsilon_{2}^{2}+\alpha_{3} \varepsilon_{2}}\end{array}\right]$.

This gives the relative dependent point of the curve

$X=X(T)=\frac{-\alpha_{4} t^{3}}{\alpha_{0} t^{3}+\alpha_{2} t^{2}+\alpha_{3} t+\alpha_{1}}$

$Y=Y(T)=\frac{-\alpha_{4} t^{2}}{\alpha_{0} t^{3}+\alpha_{2} t^{2}+\alpha_{3} t+\alpha_{1}}$

By using equation (14) and dividing by $\varepsilon_{1}$,

$\alpha_{0} \varepsilon_{2}^{3}+\alpha_{1}+\alpha_{2} \varepsilon_{2}^{2}+\alpha_{3} \varepsilon_{2}+\alpha_{4} \frac{\varepsilon_{2}^{2}}{\varepsilon_{1}}=0$.

So, by taking shadow for both sides, yield

$$
\left(\frac{\varepsilon_{2}^{2}}{\varepsilon_{1}}\right)={ }^{\circ}\left(\frac{-\alpha_{1}}{\alpha_{4}}\right)
$$

Where (20) represents a concavity in the given theory. iii - If $\Delta>0$, we have the following theorem :

Theorem 3.4: If $a_{7}=0$ and $\Delta>0$, the curve gives two tangents at regular zero point and relative zero point.

Proof : Curve tangents at the original point included two vectors, after the change coordinates for equation written

$f(x, y)=\beta_{0} x^{3}+\beta_{1} y^{3}+\beta_{2} x^{2} y+\beta_{3} x y^{2}+\beta_{4} x^{2}$

$$
+\beta_{5} y^{2} \ldots \ldots(21)
$$

If $M \in m(0)$ and $M=\varepsilon_{1} v_{1}+\varepsilon_{1} \varepsilon_{2} v_{1}=\left(\varepsilon_{1} \varepsilon_{2}, \varepsilon_{1}\right)=(\alpha, \beta)$, then

${ }^{\circ} f(M)=\beta_{4} \alpha^{2}+\beta_{5} \alpha \beta^{2}$.

Notice that the two vectors at the original point are

$v_{1}=\left[\begin{array}{l}0 \\ 1\end{array}\right]$ and $v_{2}=\frac{1}{\sqrt{\beta_{4}^{2}+\beta_{5}^{2}}}\left[\begin{array}{c}\beta_{5} \\ -\beta_{4}\end{array}\right]$

If the tangent vector perpendicular is, then $M=\left[\begin{array}{c}\varepsilon_{1} \varepsilon_{2} \\ \varepsilon_{1}\end{array}\right]$, and

$f(M)=\beta_{0} \varepsilon_{1} \varepsilon_{2}^{3}+\beta_{1} \varepsilon_{1}+\beta_{2} \varepsilon_{1} \varepsilon_{2}^{2}+\beta_{3} \varepsilon_{1} \varepsilon_{2}+\beta_{4} \varepsilon_{2}^{2}+\beta_{5} \varepsilon_{2}$ $=0 \ldots(24)$

Divide equation (24) by $\varepsilon_{1}$ to get

$\beta_{0} \varepsilon_{2}^{3}+\beta_{1}+\beta_{2} \varepsilon_{2}^{2}+\beta_{3} \varepsilon_{2}+\beta_{4} \frac{\varepsilon_{2}^{2}}{\varepsilon_{1}}+\beta_{5} \frac{\varepsilon_{2}}{\varepsilon_{1}}=0$

By taking the shadow for both sides of the above equation, yield obtain $\left(\frac{\varepsilon_{2}}{\varepsilon_{1}}\right)={ }^{\circ}\left(\frac{-\beta_{1}}{\beta_{5}}\right)$

Thus, the first curve is regular and concave at zero, that is $k=\left|\frac{\varepsilon_{2}}{\varepsilon_{1}}\right|=\left|\frac{\beta_{1}}{\beta_{5}}\right|$...

From the equation (24), $\varepsilon_{1}$ became

$\varepsilon_{1}=\frac{-\beta_{4} \varepsilon_{2}^{2}+\beta_{5} \varepsilon_{2}}{\beta_{0} \varepsilon_{2}^{3}+\beta_{2} \varepsilon_{2}^{2}+\beta_{3} \varepsilon_{2}+\beta_{1}}$

So , the curves $M$ which are

$M=\left[\begin{array}{c}\frac{-\beta_{4} \varepsilon_{2}^{3}+\beta_{5} \varepsilon_{2}^{2}}{\beta_{0} \varepsilon_{2}^{3}+\beta_{2} \varepsilon_{2}^{2}+\beta_{3} \varepsilon_{2}+\beta_{1}} \\ -\beta_{4} \varepsilon_{2}^{2}+\beta_{5} \varepsilon_{2} \\ \frac{\beta_{0} \varepsilon_{2}^{3}+\beta_{2} \varepsilon_{2}^{2}+\beta_{3} \varepsilon_{2}+\beta_{1}}{1}\end{array}\right]$

And this gives the relative correlation of the first carve

$$
M=\left[\begin{array}{l}
X(t) \\
Y(t)
\end{array}\right]=\left[\begin{array}{c}
\frac{-\beta_{4} t_{2}^{3}+\beta_{5} t_{2}^{2}}{\beta_{0} t_{2}^{3}+\beta_{2} t_{2}^{2}+\beta_{3} t_{2}+\beta_{1}} \\
\frac{-\beta_{4} t_{2}^{2}+\beta_{5} t_{2}}{\beta_{0} t_{2}^{3}+\beta_{2} t_{2}^{2}+\beta_{3} t_{2}+\beta_{1}}
\end{array}\right] .
$$

\section{References}

Daivs M. (1977), Applied Nonstandard Analysis. New York, John Wiley and Sons.

Diener M. and Lobry C. (1995), Analyse Nonstandard of Representation to Real. CNRS. , Paris.

Diener F. and Reeb G. (1989). Analyse Nonstandard. Herman, Paris.

Diener F. and Diener M . (1995), Nonstandard Analysis in Practice, Spriger-Verleg, Berlin, Heidelberg.

Henson C. W. (1997), Foundation of Nonstandard Analysis - A Gentle Introduction to Nonstandard Analysis Extension in Nonstandard Analysis : Theory and Application. ed. by N. J. Cultand and L. Arkeryd, Kluwer Academic Publishers.

Habacek K. (1979), Nonstandard Set Theory. Amer, Math. Monthly, Vol.86, No.8, pp. 659-677

Nelson E. (1977), Internal Set theory : a New Approach to Nonstandard Analysis, Bull. of Amer. Math. Sco. Vol. 83, No. 6, pp. 1165-1198.

Robinson A. (1970), Nonstandard Analysis- 2ed . American Elsevier, New-york.

Rosinger E. E. (2004), Short Introduction to Nonstandard Analysis, [arXiv : Math. GM/0407178v1,10].

Stroyan K. D. and Luxemburg W. A. (1976), Introduction to the Theory of Infinitesimal, New-York, Academic Press.

Vath M. (2007), Nonstandard Analysis , Birkhauser Verlag, Berlin. 